\title{
Advances in quasi-periodic and large commensurate systems ${ }^{\star}$
}

\author{
Tobias Stauber ${ }^{\mathrm{a}}$ and Sigmund Kohler \\ Instituto de Ciencia de Materiales de Madrid, CSIC, 28049 Madrid, Spain
}

Received 31 March 2020

Published online 11 May 2020

(C) EDP Sciences / Società Italiana di Fisica / Springer-Verlag GmbH Germany, part of Springer Nature, 2020

Non-commensurate or quasi-periodic systems attract much attention as they show interesting new phenomena and emergent phases that have been implemented in recent experiments. In general, they cannot be described by usual Bloch theory - or in the time domain: Floquet theory - and often require new approaches. Even if a system can be approximated by a commensurate one, the necessary size of a unit cell of the super lattice may quickly reach the point at which a conventional numerical treatment becomes practically impossible.

Via the tear-and-stack method, the controlled misalignment of two-dimensional van der Waals heterostructures has become possible, now, which opens up new possibilities to produce artificial incommensurate Moiréstructures. Especially twisted bilayer graphene has triggered immense research activity due to the recently observed superconductivity and correlated phases. At the so-called magic angle of $1.05^{\circ}$, the bands at neutrality become nearly flat and the unit cell already consists of approximately $10^{4}$ atoms. By decreasing the angle even further, this number can quickly reach more than $10^{5}$ atoms, making a traditional Bloch approach increasingly difficult. Even more importantly, by adding a third or further misaligned layers even a commensurate system would become virtually incommensurate or quasi-periodic.

Also in the time domain, quasi-periodicity plays a role mainly for bichromatically driven systems or, even more general, for multi-frequency driving. Then for commensurable frequencies, the signal generally depends on the phases between the frequencies of the components of the driving. For quasi-periodicity this feature usually gets lost and often the higher symmetry of the undriven system is restored. The differences between the commensurable and the non-commensurable case may manifest themselves only on rather long time scales.

This Topical Issue thus focuses on non-commensurate and quasi-periodic systems in various physical settings such as 2D Moiré lattices, quasi-periodic optical lattices,

\footnotetext{
* Contribution to the Topical Issue "Advances in QuasiPeriodic and Non-Commensurate Systems", edited by Tobias Stauber and Sigmund Kohler.

${ }^{a}$ e-mail: tobias.stauber@csic.es
}

quantum chaos and stochastic processes, or quantum systems driven with two or more frequencies.

An important class of quasi-periodic systems is generated by Fibonacci numbers as they are closely related to the most irrational of all irrational numbers, $\phi=(\sqrt{5}+$ 1) $/ 2$, the Golden Ratio. In this respect, Jagannathan and Tarzia discuss the re-entrance and localization phenomena in disordered Fibonacci chains [1]. De Dios-Leyva et al. are analysing the magneto-optical properties of Fibonacci superlattices in graphene [2]. And a Hamiltonian model of the Fibonacci quasicrystal using non-local interactions is simulated by Sen and Castro Perelman [3].

The Golden Ratio is also discussed in the analysis of the response of graphene to bichromatic driving by Kohler and Stauber [4]. Non-diagonal disorder driven by timeperiodic fields forms the subject of Molina and Gopar's research [5], whereas the generic shape of multichromatic resonance peaks is discussed by Olivera-Atencio et al. [6]. Moreover, probabilistic motional averaging is approached and outlined by Karpov et al. [7].

The work by Massatt et al. [8] develops an efficient way to compute the Kubo conductivity for incommensurate $2 \mathrm{D}$ heterostructures in which the authors go beyond the traditional Bloch approach. The interplay of a charge density wave and superconductivity in transition metal dichalcogenides is in the focus of Koley, Mohanta and Taraphder's work [9]. And last but not least, Didov et al. [10] investigate transport through degenerate tori and quantum-to-classical crossover in a driven Aubry-André model.

The main aim of this Topical Issue is to bring different scientific communities together such that recent technical developments and approaches are brought to the attention of researcher in otherwise rather different fields. Leading experts have contributed to this collection of articles with a variety of different topics and approaches. We hope that it will be inspiring and helpful for the further investigation of non-commensurate and quasi-periodic systems.

\section{References}

1. A. Jagannathan, M. Tarzia, Eur. Phys. J. B 93, 46 (2020) 
2. M. de Dios-Leyva, M.A. Hernández-Bertrán, V. Akimov, J.A. Vinasco, A.L. Morales, C.A. Duque, Eur. Phys. J. B 93, 47 (2020)

3. A. Sen, C. Castro Perelman, Eur. Phys. J. B 93, 67 (2020)

4. S. Kohler, T. Stauber, Eur. Phys. J. B 93, 24 (2020)

5. R.A. Molina, V.A. Gopar, Eur. Phys. J. B 93, 76 (2020)

6. M.L. Olivera-Atencio, J. Casado-Pascual, S. Kohler, Eur. Phys. J. B 93, 30 (2020)
7. D.S. Karpov, V.Y. Monarkha, D. Szombati, A.G. Frieiro, A.N. Omelyanchouk, E. Il'ichev, A. Fedorov, S.N. Shevchenko, Eur. Phys. J. B 93, 49 (2020)

8. D. Massatt, S. Carr, M. Luskin, Eur. Phys. J. B 93, 60 (2020)

9. S. Koley, N. Mohanta, A. Taraphder, Eur. Phys. J. B 93, $77(2020)$

10. A.A. Didov, L.E. Kon'kov, D.V. Makarov, Eur. Phys. J. B 93, $13(2020)$ 